07

\title{
О распределении носителей заряда в ветви термоэлектрического охладителя
}

\author{
(C) О.И. Марков \\ Орловский государственный университет им. И.С. Тургенева, \\ 302026 Орел, Россия \\ e-mail: O.I.Markov@mail.ru
}

Поступило в Редакцию 20 марта 2021 г.

В окончательной редакции 28 апреля 2021 г.

Принято к публикации 17 мая 2021 г.

Проведено моделирование процесса преобразования энергии в ветви термоэлектрического охладителя. Рассмотрено влияние различных распределений активной примеси вдоль ветви термоэлемента на ее эффективность в режиме максимального перепада температуры. Расчет проведен в рамках двухзонной стандартной модели зонной структуры полупроводника для невырожденных носителей заряда. Эффект Томсона не учитывался.

Ключевые слова: термоэлектрический охладитель, распределенный эффект Пельтье, двухзонная модель, невырожденные носители заряда, термоэлектрическая эффективность.

DOI: 10.21883/JTF.2021.11.51534.70-21

\section{Введение}

Основное требования к термоэлектрикам - возможно более высокое значение параметра термоэлектрической эффективности $Z=\alpha^{2} / \rho \chi$. Поэтому все старания специалистов направлены на повышение параметра $Z$. Достаточно давно появилось понимание того, что в термоэлектрических материалах необходимо создание оптимальной концентрации. Оптимизация параметра $Z$ для однозонного полупроводника проводилась еще в работе [1] и по сути дела сводилась к максимизации фактора мощности $\alpha^{2} \sigma$. Поскольку практически использующиеся термоэлектрики это легированные полупроводники $n$ - или $p$-типа, то обычно при оптимизации учитываются носители только одного знака, которые являются основными. Существуют, по крайней мере, две возможности повышения эффективности с помощью легирования: это оптимизация параметра эффективности в рабочем интервале температуры [2] и использование распределенного эффекта Пельтье [3]. В этих и других работах, посвященных оптимизации носителей заряда, учитываются носители заряда только одного знака. Обычно предполагается, что их концентрацию можно изменять с помощью легирования соответствующими примесями в очень широком интервале, не учитывая собственные носители заряда. При этом возникает желание полностью компенсировать теплоту Джоуля с помощью распределенного эффекта Пельтье [3,4]. Как показано в работе [3], в оптимизированной ветви с полной компенсацией теплоты Джоуля электропроводность на холодном конце ветви на два порядка больше чем на горячем, а модуль дифференциальной термоэдс меньше в 4 раза. В работе [5] для поддержания оптимального значения $Z$ в РbТе используются только электроны, плотность которых определяется концентрацией легиру- ющей примеси. Изменением состава материала по длине ветви термоэлемента рекомендовано поддерживать [6] показатель эффективности, близкий к максимуму в каждой точке ветви при заданной разности температур. В обзоре [7] указывается, что определение оптимального профиля неоднородности примеси является сложной математической задачей, перечисляются некоторые методы расчета температурного профиля ветви термоэлемента и рассматриваются технологии создания неоднородностей.

Реальные низкотемпературные термоэлектрики с высокой эффективностью не обладают широкой запрещенной зоной и поэтому необходимо учитывать и собственные носители заряда [8]. В настоящей работе на основе двухзонной модели термоэлектрика с использованием классической статистики сделана попытка расчета вклада распределенного эффекта Пельтье в эффективность ветви термоэлемента на основе двухзонной модели при различных видах распределения примесей. В связи с тем, что в в настоящей работе ставилась сравнительная задача действий распределенного эффекта Пельтье, эффект Томсона в решении граничной задачи не рассматривался.

\section{1. Общая схема расчета}

При оптимизации параметра термоэлектрической эффективности изменением концентрации носителей заряда основной прирост происходит в основном за счет изменений дифференциальной термоэдс и электропроводности. Из условия [1]

$$
\Delta\left(\alpha^{2} \sigma\right)>0
$$

следует, что относительное приращение электропроводности должно быть больше чем удвоенное относитель- 
ное уменьшение термоэдс:

$$
\Delta \sigma / \sigma>-2 \Delta \alpha / \alpha
$$

Учитывая, что

$$
n=N_{c} \exp \eta
$$

и формулу Писаренко

$$
\alpha=-(k / e)(r+2-\eta),
$$

можно получить оценку ограничения области приведенного химического потенциала, где возможен прирост термоэлектрической эффективности

$$
\eta<r .
$$

Таким образом, повышение эффективности преобразования при $r=-1 / 2$ химический потенциал должен лежать ниже дна зоны проводимости на расстоянии не менее $k T / 2$.

Ограничимся случаем невырожденных носителей заряда и простейшей стандартной зонной моделью. Чтобы расчеты термоэлектрика не были бы слишком абстрактными, зонные параметры приближены к параметрам реального низкотемпературного термоэлектрика на основе теллуридов висмута и сурьмы: ширина запрещенной зоны $E_{g}=0.15 \mathrm{eV}$, эффективные массы плотности состояний электронов $m_{n}^{*}=0.45 m_{0}$, дырок $m_{p}^{*}=0.69 m_{0}$ [9].

В классическом приближении концентрация электронов определяется выражением [10]:

$$
n=N_{C} \exp \eta_{e}
$$

дырок

$$
p=N_{V} \exp \eta_{h},
$$

где $N_{C}, N_{V}$ - эффективные плотности состояний зоны проводимости и валентной зоны соответственно. Концентрации электронов и дырок связаны уравнением электронейтральности:

$$
n=p+N_{d},
$$

где $N_{d}$ - концентрация донорной примеси.

Удельные электропроводности электронов $\sigma_{e}=e N_{e} n$ и дырок $\sigma_{h}=e N_{h} p$ в сумме определяют полную удельную электропроводность

$$
\sigma=\sigma_{e}+\sigma_{h} .
$$

Для лучшего сопоставления результатов расчета с экспериментом необходимо учесть температурные зависимости подвижностей носителей заряда и решеточной теплопроводности. В достаточно широком температурном интервале можно учесть температурную зависимость подвижности носителей заряда в виде $U_{e}=617.3 \cdot T^{-3 / 2}, U_{h}=1449 \cdot T^{-1 / 8}[9,11]$. Концентрационные зависимости подвижностей электронов и дырок не учитывались.
Удельная теплопроводность полупроводника

$$
\chi=\chi_{p h}+2\left(\frac{k}{e}\right)^{2} T\left[\sigma_{e}+\sigma_{h}+\frac{\sigma_{e} \sigma_{h}}{\sigma_{e}+\sigma_{h}}\left(\frac{E_{g}}{k T}+4\right)^{2}\right],
$$

где $\chi_{p h}-$ фононная теплопроводность, решеточная составляющая теплопроводности учтена в виде зависимости $\chi_{p h}=235 \cdot T^{-1}[9]$.

Дифференциальная термоэдс равна [9]

$$
\alpha=\frac{\alpha_{p} \sigma_{p}+\alpha_{n} \sigma_{n}}{\sigma_{p}+\sigma_{n}},
$$

где парциальные термоэдс электронов и дырок определяются формулой Писаренко (4).

Оптимизация параметра эффективности $Z=\alpha^{2} / \rho \chi$ при $T=300 \mathrm{~K}$ показывает, что максимальное значение $3 \cdot 10^{-3} \mathrm{~K}^{-1}$ достигается при значении приведенного химического потенциала $\eta=-0.4$ и концентрации примеси $N_{d}=5 \cdot 10^{24} \mathrm{~m}^{-3}$. Согласно элементарной теории А.Ф. Иоффе $\Delta T=Z T_{c}^{2} / 2$ [1], можно рассчитать минимально достижимую на холодном конце ветви температуру $-225 \mathrm{~K}$. Расчет при такой температуре дает параметр эффективности $Z=2.95 \cdot 10^{-3} \mathrm{~K}^{-1}$ при значении химического потенциала $\eta=-0.5$ и концентрации примеси $N_{d}=3 \cdot 10^{24} \mathrm{~m}^{-3}$. Естественно, возникает вопрос: какова же должна быть оптимальная концентрация примеси в однородной ветви? Для ответа на него необходимо воспользоваться решением граничной задачи теплового баланса:

$$
\frac{d}{d \xi}\left(\chi \frac{d T}{d \xi}\right)+\frac{Y^{2}}{\sigma}=0
$$

с граничными условиями

$$
\left.\chi \frac{d T}{d \xi}\right|_{\xi=0}=\left.\alpha Y T\right|_{\xi=0},\left.T\right|_{\xi=1}=T_{1},
$$

где $\xi=x / l(0<\xi<1), Y=I l / S$ параметр оптимизации $[12], l-$ длина ветви, $S-$ сечение ветви, $I-$ ток ветви. Поскольку граничная задача нелинейная, она решалась численными методами. Минимизация температуры холодного конца ветви по параметру $Y$ с одновременной оптимизацией по концентрации примеси дала значение $230 \mathrm{~K}$. Оптимальная концентрация примеси для однородной ветви оказалась равной $N_{d}=3.3 \cdot 10^{24} \mathrm{~m}^{-3}$. На рис. 1 отражено распределение приведенного химического потенциала вдоль ветви (кривая 3), что в целом удовлетворяет условию (5), но выходит за границы рассчитанного выше интервала химического потенциала, соответствующие максимумам $Z$ при температурах 230 и $300 \mathrm{~K}$. Поэтому эффективно работает только небольшая часть ветви вблизи ее холодного конца. Это однозначно указывает на то, что оптимальной по параметру $Z$ может быть только неоднородная по составу ветвь. Каков же должен быть характер неоднородности? Это 


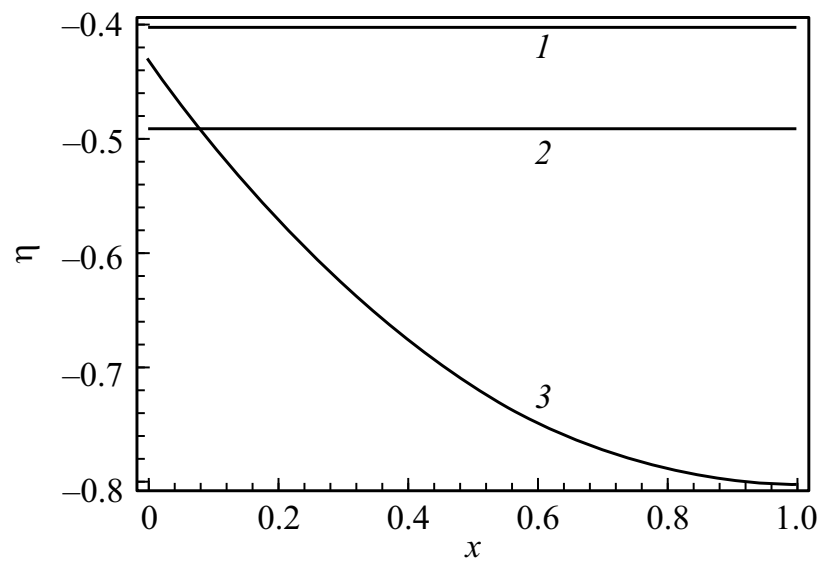

Рис. 1. Распределение химического потенциала вдоль однородной ветви с оптимальной концентрацией примеси (кривая 3); прямые 1 и 2 отвечают оптимальным значениям химического потенциала при 300 и $225 \mathrm{~K}$ соответственно.

можно определить, решая граничную задачу теплового баланса с учетом распределенного эффекта Пельтье:

$$
\frac{d}{d \xi}\left(\chi \frac{d T}{d \xi}\right)+\frac{Y^{2}}{\sigma}-Y T \frac{d \alpha}{d n} \frac{d n}{d \xi}=0
$$

и прежними граничными условиями (13). Поскольку имеется аналитическая зависимость между концентрацией электронов и концентрацией примеси

$$
n\left(N_{d}\right)=\frac{N_{d}}{2}\left(\sqrt{1+\frac{4 n_{i}^{2}}{N_{d}}}+1\right)
$$

производную от концентрации электронов можно выразить через производную от концентрации примеси

$$
\frac{d n}{d \xi}=\frac{d n}{d N_{d}} \frac{d N_{d}}{d \xi},
$$

что позволяет решать граничную задачу (13), (14) при различных видах распределения примеси вдоль ветви $N_{d}(\xi)$.

Решая задачу (13), (14) с линейным распределением концентрации примеси в рассчитанных выше пределах от $N_{d}(0)=3 \cdot 10^{24} \mathrm{~m}^{-3}$ на холодном конце до $N_{d}(1)=5 \cdot 10^{24} \mathrm{~m}^{-3}$ на горячем с одновременной оптимизацией по параметру $Y$, получим, что вместо ожидаемого понижения минимально достижимой температуры на холодном конце ветви, она повышается до $234 \mathrm{~K}$, что связано с выделением теплоты за счет распределенного эффекта Пельтье. Как видно из рис. 2, отличие концентрации электронов от концентрации примеси невелико, потому что концентрация собственных электронов на порядок меньше. На вставке (рис. 2) видно, что в этом случае положение приведенного химического потенциала лучше удовлетворяет условию (5). Таким образом, в случае оптимизации параметра $Z$ по температуре вдоль ветви для расчетов иногда достаточно однозонного приближения. Распределение приведенной плотности теплот $q l / S$ Джоуля и распределенного эффекта Пельтье приведено на рис. 3. Неоднородность ветви приводит к перераспределению плотности теплоты Джоуля и поскольку удельное сопротивление вблизи холодного конца ветви выше, то максимум количества теплоты Джоуля смещается к холодному концу ветви (кривая 1). Свой негативный вклад вносит и распределенный эффект

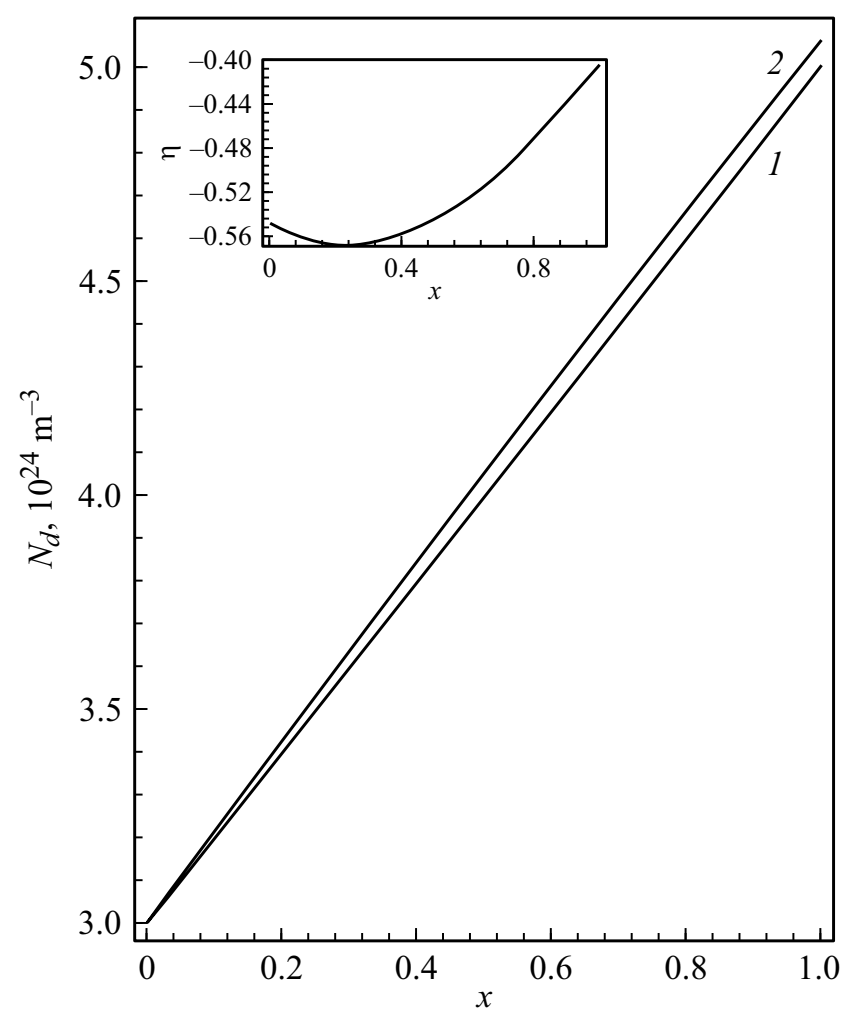

Рис. 2. Распределение концентраций примеси (1) и электронов $n(2)$ вдоль ветви (на вставке распределение химического потенциала вдоль ветви).

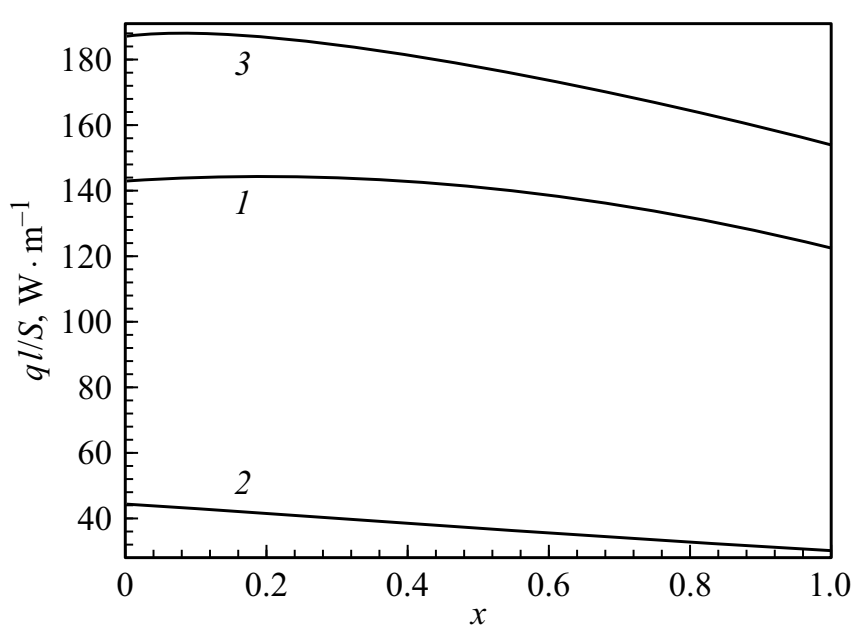

Рис. 3. Распределение приведенной плотности теплот Джоуля (1), распределенного Пельтье (2) и их суммы (3). 


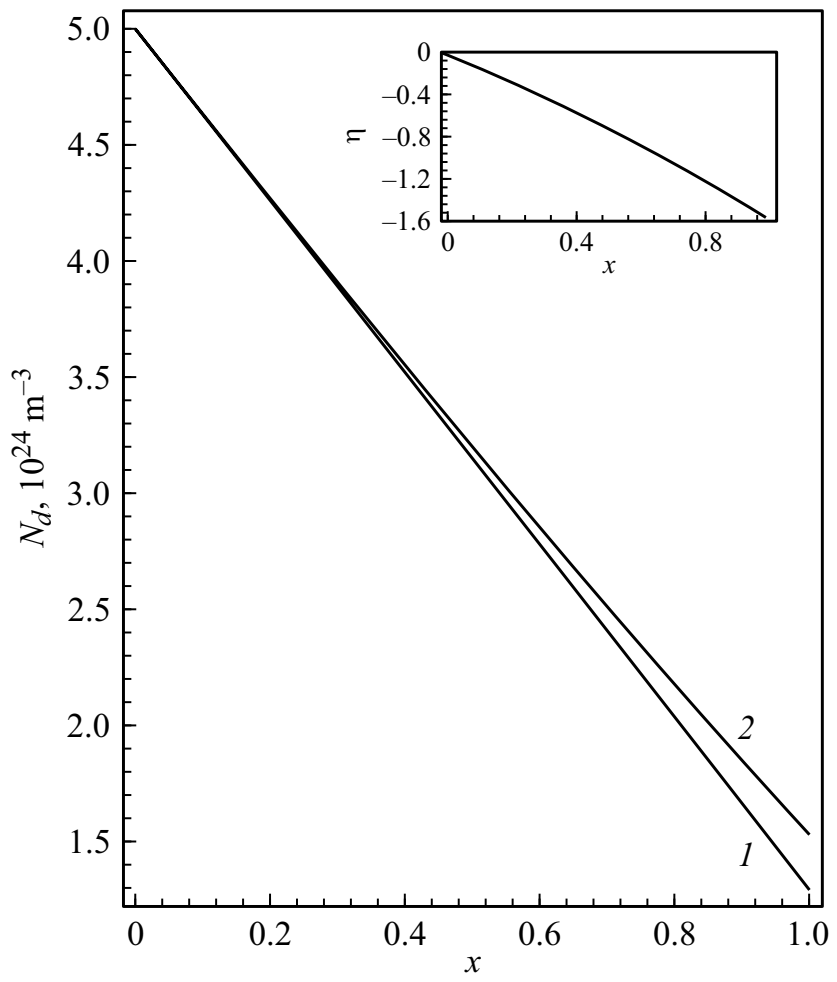

Рис. 4. Распределение концентраций примеси (1) и электронов $n(2)$ вдоль ветви (на вставке распределение химического потенциала вдоль ветви).

Пельтье (кривая 2), в результате чего общий максимум тепловыделения приближается к холодному концу ветви (кривая 3). Поэтому оптимизация параметра $Z \mathrm{c}$ целью повышения термоэлектрической эффективности по температуре оказывается нерезультативна. Оценка вклада приведенной теплоты Томсона вблизи холодного конца ветви, где градиент температуры наибольший, дает величину, не превышающую $16 \mathrm{~W} / \mathrm{m}$.

Повышение эффективности термоэлектрического преобразования энергии с использованием распределенного эффекта Пельтье не сводится только к оптимизации параметра эффективности $Z$, но кроме достаточно высоких значений $Z$ еще требуется и максимально увеличить диапазон изменения модуля дифференциальной термоэдс. Однако это расширение области со стороны больших концентраций носителей заряда ограничено падением модуля термоэдс из-за вырождения носителей заряда, а верхняя граница связана с максимально достижимым значением модуля дифференциальной термоэдс, который может быть определен только в рамках двухзонной модели. Для проведения расчета в этом случае необходимо решение граничной задачи (13), (14). Расчет термоэдс показал, что максимальное значение модуля при $300 \mathrm{~K}$ достигается при концентрации примеси $N_{d}(1)=1.3 \cdot 10^{24} \mathrm{~m}^{-3}$. Выбор концентрации примеси на холодном конце ветви был сделан в результате перебора разных значений в серии решений граничной задачи с использованием линейного распределения. Выяснилось, что максимальный перепад температур достигался при линейном распределении примеси от $N_{d}(0)=5 \cdot 10^{24} \mathrm{~m}^{-3}$ на холодном конце до $N_{d}(1)=1.3 \cdot 10^{24} \mathrm{~m}^{-3}$ на горячем. Следует заметить, что ни на холодном конце ветви, ни на горячем концентрация примеси не является оптимальной для максимального $Z$ при данной температуре. Рассчитанное распределение концентрации электронов (15) представлено на рис. 4. В данном случае отличие от концентрации примеси несколько больше чем в предыдущем случае, поскольку увеличивается вклад собственных носителей на горячем конце ветви. При таком распределении примеси в ветви происходит существенное перераспределение теплот Джоуля и Пельтье, что и способствует повышению эффективности (рис. 5).

Минимально достижимая температура на холодном конце ветви при таком распределении примеси составляет $226 \mathrm{~K}$, т.е. практически совпадает с расчетом по теории А.Ф. Иоффе. Модуль дифференциальной термоэдс от холодного к горячему концу увеличивается лишь в 1.5 раза, а удельное сопротивление в 4.8 раз, что существенно отличается от данных расчетов для однозонного термоэлектрика в работе [4]. На основании проделанных расчетов можно сделать вывод, что возлагать надежду на полную компенсацию теплоты Джоуля в низкотемпературном термоэлектрике не следует. Поскольку в граничной задаче (13), (14) линеаризация не проводилась и соответственно использование развитых методов оптимизации [4] при решении нелинейной задачи невозможно, то остается лишь ограничиться подбором вида распределения примеси, добиваясь максимального снижения температуры. Нахождение же оптимального распределения примеси является достаточно сложноосуществимой задачей, которую, по-видимому, еще сложнее воплотить технологически.

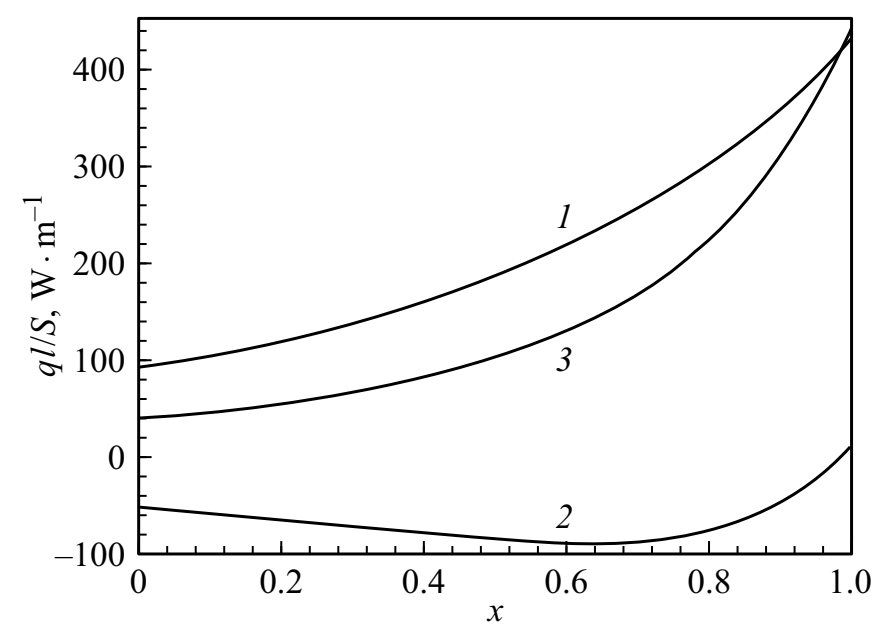

Рис. 5. Распределение приведенной плотности потоков теплот Джоуля (1), распределенного Пельтье (2) и их суммы (3). 


\section{Заключение}

С использованием двухзонной модели термоэлектрика решена граничная тепловая задача для ветви термоэлемента. На примерах различных распределений примеси рассчитаны температурное поле, приведенный химический потенциал, распределение тепловыделений. Такой подход позволил детально проанализировать влияние распределенного Пельтье на эффективность термоэлектрического охлаждения.

Проведенное исследование позволяет заключить, что:

— в рамках двухзонной стандартной модели зонной структуры термоэлектрика для повышения эффективности ветви $n$-типа можно использовать неоднородное распределение концентрации донорной примеси;

- оптимизация концентрации носителей для создания максимальных значений $Z$ на холодном и горячем концах и линейного распределения концентрации донорной примеси вдоль ветви не приводит к повышению ее эффективности из-за повышения тепловыделений эффекта Джоуля и распределенного эффекта Пельтье вблизи холодного конца ветви;

- использование линейного распределения концентрации донорной примеси позволяет повысить эффективность преобразования ветви как в результате частичной компенсации тепловыделения эффекта Джоуля за счет распределенного эффекта Пельтье, так и в результате перераспределения плотности тепловыделения Джоуля к горячему концу ветви;

- использование распределенного эффекта Пельтье для повышения эффективности ограничено интервалом достаточно высоких значений модуля дифференциальной термоэдс, что со стороны наибольшей концентрации примеси обусловлено вырождением носителей заряда и появлением вклада собственных носителей заряда для наименьшей концентрации;

- оптимальное распределение примеси вдоль ветви однозначно связано с ее температурным профилем и любое изменение тепловой нагрузки ветви делает его неоптимальным, поэтому ее расчет является скорее теоретической задачей, чем практической проблемой.

\section{Конфликт интересов}

Автор заявляет, что у него нет конфликта интересов.

\section{Список литературы}

[1] А.Ф. Иоффе. Полупроводниковые термоэлементы (АН СССР, М.-Л., 1960)

[2] Е.К. Иорданишвили. ФТТ, 8 (10), 3118 (1966).

[3] Ю.И. Агеев, К.Ф. Иванова, М.А. Каганов, Л.С. Стильбанс, Э.М. Шер. ЖТФ, 55 (11), 2266 (1985).

[4] К.Ф. Иванова, А.С. Ривкин. ЖТФ, 52 (7), 1406 (1982).

[5] S. Dashevsky, S. Shusterman., M.P. Dariel, I. Drabkin. J. Appl. Phys., 92 (3), 1425 (2002).
[6] A.E. Kaliazin, V.L. Kuznetsov, D.M. Rowe. Proc. of the 20th Int. Conf. on Thermoelectrics (Beijing, China, 2001), p. 286.

[7] V.L. Kuznetsov. In: CRC Thermoelectrics Handbook, ed. by D.M. Rowe (CRC Press, Boca Raton, London, NY., 2006), p. 38.

[8] О.И. Марков. Докл. ХІІІ Межггосударственного семинара „Термоэлектрики и их применения“ (СПб., Россия, 2013), c. 180.

[9] Б.М.Гольцман, В.А. Кудинов, И.А. Смирнов. Полупроводниковые термоэлектрические материалы на основе $B i_{2} T_{3}$ (Наука, М., 1972)

[10] Б.И. Аскеров. Электронные явления переноса в полупроводниках (Наука, М., 1985)

[11] Термоэлектрические охладители, под ред. А.Л. Вайнера. (Радио и связь, М., 1983)

[12] О.И. Марков. ЖТФ, 75 (6), 132 (2005). 